\title{
Geodesic Line on the Surface of a Spheroid
}

\author{
By Roger Bourbon \\ (Inspecteur Général de l'enseignement maritime (Retd.))
}

T. Hiraiwa, as well as R. Williams and J. E. Phythian, explain, in volumes 40 and 42 of the Journal of Navigation, methods of calculating the length of a geodesic line between two far points of a spheroid.

I read these two articles with great interest and I intend to give below another method of calculating this length. My method is based partly on the 'notice scientifique' of $H$. Andoyer, published in 1932 in issue 34 of the 'Bulletin Géodésique' and quoted by the three authors. This article was written when computations were only worked out with logarithm tables. At that time, it was necessary to look for methods avoiding tedious calculations. Therefore, from the article of $\mathrm{H}$. Andoyer, I shall principally retain the true formulae and the very general methods of calculating integrals that are not expressed with elementary functions.

In this article, the author represents by

$e$ the eccentricity of the Earth;

$\alpha$ the oblateness of the Earth;

$\phi, l$ the geodesic latitude and longitude of a point of the Earth,

$\psi$ the eccentric latitude $[\operatorname{tg} \psi=(1-\alpha) \operatorname{tg} \phi]$

$p$ a constant that is the particular value of $\psi$ corresponding to the latitude $\phi_{f}$ of a geodesic line's vertex $\left[p=\psi_{v}\right]$.

He also specifies that along a geodesic line, we have:

$$
\begin{gathered}
\sin \psi=\sin p \sin u, \quad \cos p \operatorname{tg} u=\operatorname{tg} v, \\
d L=d v+\cos p\left(\frac{\sqrt{ }\left(1-e^{2} \cos ^{2} \psi\right)-1}{\cos ^{2} \psi}\right) d u
\end{gathered}
$$

and that the length $\sigma$ of a geodesic line with the equatorial radius taken as the unit is:

$$
d \sigma=\sqrt{ }\left(1-e^{2} \cos ^{2} \psi\right) d u{ }^{1}
$$

In fact, the formulae giving $d L$ assumes that the longitude (counted positively towards the East) and the latitude vary in the same way and the formula giving $d \sigma$ assumes that the geodesic line is positively orientated in the direction of increasing latitudes.

Let $M$ be a point of a geodesic line and let $\gamma$ be the angle between the meridian of $M$ and the geodesic line orientated from the point of departure $D$ to the point of arrival $A$. The angle $\gamma$ is counted from o to $2 \pi$ and from North to East. As it is convenient to use an algebraic formula to obtain the longitude and a formula always giving a positive value of the length, it is better to let $\epsilon=\epsilon^{\prime}= \pm 1$ and to write:

$$
\epsilon d L=d v+\cos p\left(\frac{\sqrt{ }\left(1-e^{2} \cos ^{2} \psi\right)-1}{\cos ^{2} \psi}\right) d u, \quad \epsilon^{\prime} \mathrm{d} \sigma=\sqrt{ }\left(\mathrm{I}-e^{2} \cos ^{2} \psi\right) d u
$$


The values of $\epsilon$ and $\epsilon^{\prime}$ are given by the following table:

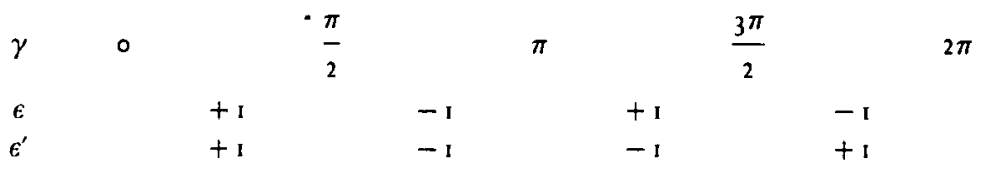

A passage through a vertex involves a change of sign of formulae giving $d L$ and $d \sigma$. H. Andoyer also shows that the value of

$$
\sigma=\int_{u}^{u^{\prime}} \sqrt{ }\left(1-e^{2} \cos ^{2} \psi\right) d u=\int_{u}^{u^{\prime}} \sqrt{ }\left(1-e^{2}+e^{2} \sin ^{2} p \sin ^{2} u\right) d u
$$

can be considered as equal to:

$$
\sigma=\left(u^{\prime}-u\right)\left(1-\alpha+\frac{\alpha}{2} \sin ^{2} p\right)-\frac{\alpha}{4} \sin ^{2} p\left(\sin 2 u^{\prime}-\sin 2 u\right),
$$

where $u$ is the value corresponding to the latitude $\phi$ of the point of departure $D$ and $u^{\prime}$ the value corresponding to the latitude $\phi^{\prime}$ of the point of arrival $A$.

In fact, the value of $\sigma$ given by ( 1 ) is an approximate value obtained when limiting to its first two terms the development of $\sqrt{ }\left(1-e^{2}+e^{2} \sin ^{2} p \sin ^{2} u\right)$ and taking $e^{2}=2 a$.

If this last approximation is not made but if we take into account the first three terms of the development of $\sqrt{ }\left(1-e^{2} \cos ^{2} \psi\right)$ while neglecting, after integration, the terms where the factor $e^{4} / 64=0.000000706$ i.e. $0 . " 146$ appears, we find, after having introduced the factor $\epsilon^{\prime}= \pm \mathrm{I}$ and taken $e^{2}=0.006272670$ :

$$
\epsilon^{\prime} \sigma=P\left(u^{\prime}-u\right)+Q\left(\sin 2 u^{\prime}-\sin 2 u\right) \text {, }
$$

where

$$
\begin{aligned}
& P=0.9966330+0.0016863 \sin ^{2} p \\
& Q=-0.0008432 \sin ^{2} p .
\end{aligned}
$$

We must make use of this formula (2) when the vertex $V_{x}$ of the geodesic line $D A$ is not between $D$ and $A$, the sign of $\epsilon^{\prime}= \pm 1$ being given by the direction of the geodesic line.

If the vertex $V_{x}$ of the geodesic line is between $D$ and $A$, we must use:

$$
\epsilon^{\prime} \sigma=P\left[\pi-\left(u+u^{\prime}\right)\right]-Q\left(\sin 2 u+\sin 2 u^{\prime}\right)
$$

giving to $E^{\prime}= \pm 1$ the sign deduced from the direction of the geodesic line $D V_{x}$.

Now we may wonder whether it is not sufficient to use formulae (2) or (3) to work out the length of the geodesic line.

The simple inspection of the formula (3), for instance, shows that, as $P$ is near 1 , an error in $u$ whose upper limit of the absolute value is $\Delta u$ and an error in $u^{\prime}$ whose upper limit of the absolute value is $\Delta u^{\prime}$ lead to an error in $\sigma$ whose upper limit of the absolute value $\Delta \sigma$ is near $\Delta u+\Delta u^{\prime}$.

But these errors $\Delta u$ and $\Delta u^{\prime}$ can only be the result of an error in $p$, i.e. of an error in the latitude of the vertex of the geodesic line.

We have

$$
\begin{aligned}
\sin u & =\frac{\sin \psi}{\sin p}, \quad \cos u d u=-\frac{\sin \psi}{\sin ^{2} p} \cos p d p, \\
\cos u d u & =-\frac{\sin u \sin p}{\sin ^{2} p} \cos p d p, \quad d u=\frac{-\operatorname{tg} u}{\operatorname{tg} p} d p .
\end{aligned}
$$


Thence

$$
\Delta u+\Delta u^{\prime}=\left(\frac{\operatorname{tg} u+\operatorname{tg} u^{\prime}}{\operatorname{tg} P}\right) \Delta p .
$$

If, for instance, we take the example given by R. Williams and J. E. Phythian on page 135 of volume 42 of the Journal of Navigation and relating to the geodesic line between $D\left(\phi=51^{\circ} 46^{\prime} \mathrm{N}, L=55^{\circ} 22^{\prime} \mathrm{W}\right)$ and $A\left(\phi^{\prime}=55^{\circ} 32^{\prime} \mathrm{N}, L^{\prime}=07^{\circ} 14^{\prime} \mathrm{W}\right)$, we obtain with the value of the great circle vertex latitude $\left(56^{\circ} 29^{\prime}, 6 \mathrm{~N}\right)$ as the value of the geodesic line vertex latitude: $\operatorname{tg} u=2.80141, \operatorname{tg} u^{\prime}=6.60000, \operatorname{tg} p=1.50537$. Thence, $\Delta u+\Delta u^{\prime}=6.25 \Delta p$.

An error of $I^{\prime}$ in the calculation of $p$ gives an error greater than $6^{\prime}$ in the length of the geodesic line, which is unacceptable.

The formulae (2) and (3) may only be used for the computation of the length of a geodesic line if the latitude of the vertex of this line is accurately known.

This latitude can be worked out accurately using the equation of the geodesic line obtained by integration of the differential equation given by $\mathrm{H}$. Andoyer (except for the factor $\epsilon= \pm 1$ ):

$$
\epsilon d L=d v+\cos p\left(\frac{\sqrt{ }\left(1-e^{2} \cos ^{2} \psi\right)-1}{\cos ^{2} \psi}\right) d u .
$$

If only the first four terms of the development of $\sqrt{ }\left(1-e^{2} \cos ^{2} \psi\right)$ are taken and if the terms with $\mathrm{e}^{6}$ are not taken into account, we have:

$$
\epsilon\left(L^{\prime}-L\right)=\left(v^{\prime}-v\right)+\cos p\left(A+B \sin ^{2} p\right)\left(u^{\prime}-u\right)-\frac{B}{2} \cos p \sin ^{2} p\left(\sin 2 u^{\prime}-\sin 2 u\right),
$$

where $A=-0.0033670$ and $B=0.0000028$.

It is easy to see that the maximum of $\cos p \sin ^{2} p$, obtained when $p= \pm \arcsin \sqrt{ } \frac{2}{3}$ is 0.38490 . The maximum of the absolute value of $\left(\sin 2 u^{\prime}-\sin 2 u\right)$ is 2 . Hence, the maximum of $\frac{B}{2} \cos p \sin ^{2} P\left(\sin 2 u^{\prime}-\sin 2 u\right)$ is 0.000001 corresponding to $0 . " 23$. The third term of (4) is negligible. Therefore, we may write when the vertex $V_{x}$ of the geodesic line is not between $D$ and $A$ :

$$
\epsilon\left(L^{\prime}-L\right)=\left(v^{\prime}-v\right)+\cos p\left(A+B \sin ^{2} p\right)\left(u^{\prime}-u\right)
$$

the sign of $\epsilon= \pm \mathrm{I}$ being given by the direction of this geodesic line.

If the vertex of the geodesic line is between $D$ and $A$, we have to use:

$$
\epsilon\left(L^{\prime}-L\right)=\left[\pi-\left(v+v^{\prime}\right)\right]+\cos p\left(A+B \sin ^{2} p\right)\left[\pi-\left(u+u^{\prime}\right)\right]
$$

the sign of $E= \pm \mathrm{I}$ being given by the direction of the geodesic line $D V_{x}$.

To compute $p$ one must give successive values to $\phi_{v}$ to obtain by means of (5) or (6) the known value $L^{\prime}-L$ (or a very near value).

When $P$ is determined by this process, the value of $\sigma$ is computed by means of (2) or (3).

Worked Example. Let us refer to the example given by R. Williams and J. E. Phythian on page 135 of volume 42 of the Journal of Navigation and relating to the geodesic line between $D\left(\phi=51^{\circ} 46^{\prime} \mathrm{N}, L=55^{\circ} 22^{\prime} \mathrm{W}\right)$ and $A\left(\phi^{\prime}=55^{\circ} 32^{\prime} \mathrm{N}, L^{\prime}=07^{\circ} 14^{\prime} \mathrm{W}\right)$.

The latitude and the longitude of the vertex of the great circle are $\phi_{v}=56^{\circ} 29^{\prime} 34^{\prime \prime} \mathrm{N}$, $L_{v}=22^{\circ} 32^{\prime} 33^{\prime \prime} \mathrm{W}$. 
The value of $L_{v}$ shows that the formula (6) is to be used. The value $\epsilon=+1$ is also to be taken. We have $L^{\prime}-L=48^{\circ} \circ 8^{\prime}$.

We shall attempt, by successive approximations, to obtain $L^{\prime}-L=4^{\circ} \circ 8^{\prime}$.

FIRST APPROXIMATION. We take $\phi_{v_{1}}=56^{\circ} 29^{\prime} 34^{\prime \prime}$. The formula (6) gives:

$$
\left(L^{\prime}-L\right)_{1}=48^{\circ} \circ 4^{\prime} 54^{\prime \prime} \text {. }
$$

SECOND APPROXIMATION. We take $\phi_{v_{2}}=56^{\circ} 30^{\prime} 00^{\prime \prime}$. The formula (6) gives:

$$
\left(L^{\prime}-L\right)_{2}=48^{\circ} \circ 9^{\prime} 47^{\prime \prime} \text {. }
$$

THIRD APPROXIMATION. We take

$$
\phi_{v 3}=\phi_{v 1}+\frac{\left(\phi_{v 2}-\phi_{v 1}\right) \times\left[\left(L^{\prime}-L\right)-\left(L^{\prime}-L\right)_{1}\right]}{\left(L^{\prime}-L\right)_{2}-\left(L^{\prime}-L\right)_{1}}
$$

i.e.

$$
\phi_{v 3}=56^{\circ} 29^{\prime} 50^{\prime \prime} .5 \text {. }
$$

The formula (6) gives: $\left(L^{\prime}-L\right)_{3}=48^{\circ} \circ 8^{\prime}$ o0" (exactly $4^{\circ} \circ 7^{\prime} 59^{\prime \prime} .897$ ).

We may consider that $\phi_{v 3}=56^{\circ} 29^{\prime} 50^{\prime \prime} .5$ is the true value of the latitude of the vertex of the geodesic line.

We obtain the length DA by means of the formula (3). It can be noted that this calculation is very easy as the values of $u$ and $u^{\prime}$ have been found in the third approximation.

We find $\sigma=1695^{\prime} .27$.

The length calculated by R. Williams and J. E. Phythian when taking points at $5^{\circ}$ intervals of longitude is $1695^{\prime} .21$.

To conclude, like R. Williams and J. E. Phythian, I shall leave the readers to judge whether or not the amount of work involved justifies this method as an alternative to the correction method of Andoyer or to the work of Hiraiwa or of Williams and Phythian themselves.

\section{NOTES}

I. Patently, there is a misprint at the top of page 80 of the 'Bulletin Géodésique'. We must apparently read $\psi$ and not $\phi$.

\section{KEY WORDS}

I. Geodesy. 